\title{
POKÉMON GO DID NOT INCREASE STEP COUNT OR DISTANCE TRAVELLED AMONG COLLEGE STUDENTS
}

original paper

(c) University School of Physical Education in Wroclaw

DOI: https://doi.org/10.5114/hm.2020.89916

\author{
SERENA A. SCHADE ${ }^{1}$, JOSEPH M. MAHONEY ${ }^{2,3}$, APRIL V. SPOTTS ${ }^{3}$, \\ NATHAN GREENAUER ${ }^{4}$, PRAVEEN VEERABHADRAPPA ${ }^{3}$ \\ ${ }^{1}$ Life Science, Pennsylvania State University, Berks Campus, Reading, USA \\ ${ }^{2}$ Mechanical Engineering, Pennsylvania State University, Berks Campus, Reading, USA \\ ${ }^{3}$ Kinesiology, Pennsylvania State University, Berks Campus, Reading, USA \\ ${ }^{4}$ Applied Psychology, Pennsylvania State University, Berks Campus, Reading, USA
}

\section{ABSTRACT}

Purpose. Physical inactivity is a well-known problem in the United States, especially among the younger population. Pokémon Go (PG) is a mobile game that has players catch Pokémon while walking in the real world. The purpose of this study was to assess the effect of playing PG on student physical activity.

Methods. Overall, 27 healthy undergraduate students participated in a 2-week study. They were randomly assigned to either PG-playing group or control group. Fitbit Charge HR devices were deployed for 2 weeks to all participants. The daily number of steps and distance travelled were recorded and the groups were compared by using the bootstrapped $t$-test.

Results. No statistically significant difference in means was observed when comparing the average daily step count $(p=0.845)$ or the average daily distance travelled $(p=0.528)$ between control and PG-playing participants. Furthermore, the mean difference was close to 0 for each comparison, with $95 \%$ confidence intervals of -2072 to 2438 steps and -0.609 to $1.31 \mathrm{~km}$, respectively. Conclusions. PG did not lead to a statistically observable increase in the number of steps or distance travelled by university students. These results may provide an impetus to create alternative interventions with better user engagement by applying mobile health apps with the intent to reach low-activity populations.

Key words: pedometry, e-gaming, active video gaming, exercise, health promotion

\section{Introduction}

Those who are physically active tend to live longer, perform better academically, and are at lower risk of developing various health conditions and diseases [1]. It is recommended for adults to participate in at least 2.5 hours per week of moderate-intensity exercise as well as at least 1.25 hours of vigorous activity [2]. Around $80 \%$ of U.S. adults and adolescents are not engaging in adequate amounts of physical activity [2]. Thus, it is concerning that most undergraduate students spend significant time on sedentary behaviours, sitting for 6 or more hours per day [3]. One study found that $70 \%$ of students gained approximately $9 \mathrm{lbs}$. during their first 2 years of college [4]. Since students spend significant amounts of time on their coursework, watching television, and playing console video games, there is a drift towards sedentary behaviour [5]. In recent years, there have been new mobile games that embed gameplay within the physical world, categorized as exergames or active video games. These games may increase a player's physical activity, especially in lowactivity populations [6]. One popular example of an active video game is Niantic's Pokémon Go (PG).

One aspect which makes PG unique when compared with traditional mobile games is that it encourages players to explore and to 'get up and go'. This game prompts and engages users to walk indoors and outdoors to capture and hatch virtual creatures, gain experience points, and battle other players within the game. This concept, being physically active to increase levels within the game, has the potential to raise the

Correspondence address: Joseph M. Mahoney, 225 Gaige Building, Penn State Berks, Reading, PA 19610, USA, e-mail: jmm694@psu.edu

Received: December 28, 2018

Accepted for publication: August 30, 2019

Citation: Schade SA, Mahoney JM, Spotts AV, Greenauer N, Veerabhadrappa P. Pokémon go did not increase step count or distance travelled among college students. Hum Mov. 2020;21(2):64-70; doi: https://doi.org/10.5114/hm.2020.89916. 
physical activity of the player. Additionally, PG's social and exploration aspects were seen to have a high level of value to players, which could increase adherence and encourage physical activity [7]. Active video games, such as this one, have gained momentum in society and could be potentially used as a tool to prevent sedentary behaviour and promote physical activity [6]. On the game's 1-year anniversary, in June 2017, it had reached 750 million downloads around the world [8]. This number of users gives reason to study if PG could be an effective tool to combat undergraduate sedentary behaviour.

There have been several previous studies to test the effectiveness of PG on increasing physical activity. One such study [9] found playing this game for 1 hour to be an effective way to reach the recommended levels of physical activity. Specifically, it was observed that students took 6000 steps per hour while actively playing PG. However, the study was performed in a controlled environment in which the researcher remained with the participants and stayed within a 2-mile radius. This does not reflect day-to-day conditions in which users would be playing. Another study [6] found that playing PG could increase physical activity by 30 minutes per week. However, methodological limitations in these studies raise concerns about the validity of their findings. As no control groups were applied, it is unclear whether PG had an influence on physical activity. Furthermore, some conclusions were based exclusively on self-reported estimates of physical activity [10], which typically overrate actual performance [11]. In cases where an objective measure of physical activity was used [6, 12], activity was monitored for only a short duration so little or no information was provided about mid- or long-term benefits of PG.

One study has collected objective measures of gameplay over an extended duration [12]. Specifically, the step counts of participants 4 weeks prior to downloading PG as well as during 6 weeks of playing the game were reported. An initial increase in steps per day was observed during the first week of gameplay. However, steps per day after 6 weeks were comparable with those prior to downloading PG, indicating short-lived benefits to activity. Although this study recruited a large national sample, it involved participants using only an iPhone 6 . Another study [13] found similar results of increased walking distance during the first week of playing PG, but no difference in the activity levels beyond the first week of playing. This study utilized iPhones to track walking distance and had a control group. The sample size comprised 65 students; however, participation was limited to iPhone users.
One possible benefit of PG is to encourage individuals to be more active every day; thus, it is reasonable to assess the effects of the game on physical activity for 2 weeks among college students at a local university by using an activity monitor. The aims of this study were to determine if PG players would have (1) a higher average daily step count and (2) a higher average daily walking distance when compared with nonplayers.

\section{Material and methods}

\section{Participants}

A convenience sample of 27 undergraduate students aged over 18 years at the Pennsylvania State University, Berks Campus, were recruited by using e-mail invitations and word-of-mouth announcements. The participants were randomly assigned to either the Pokémon group, who played PG for the entirety of the study, or the control group, who refrained from playing PG. The subjects were naïve to the purpose of the experiment and given no instructions about their physical activity during the data collection.

The students completed a medical history and demographic questionnaire, which allowed to assess their normal physical activity patterns and cardiac risk factors prior to the start of the study.

Three cohorts completed the protocol. The data collection for each cohort began and ended on the same day. The 3 cohorts began on November 28, 2016 (10 subjects), January 24, 2017 (11 subjects), and February 8, 2017 (6 subjects). The 3 start dates are considered 'seasons' in future analyses.

\section{Protocol}

Upon arrival, initial anthropometric measurements were collected. An Omron Bioimpedance device (model HBF-516B, Omron Healthcare, Inc., Bannockburn, USA) was used to measure the participants' weight. Fitbit Charge Heart Rate FB405BKL monitors were applied to determine step count, minutes of physical activity, caloric expenditure, and resting heart rate $[14,15]$. All subjects were provided a Fitbit Charge HR FB405BKL (Fitbit, San Francisco, USA), a daily activity log, and instructions to play PG (for those assigned to the Pokémon group). The students completed a survey containing the Revised Physical Activity Readiness Questionnaire (rPAR-Q) [16]. At the end of the 2-week period, researchers collected data from the assigned Fitbits. 


\section{Statistical analysis}

The mean number of daily steps and distance travelled were compared between the condition of participants that did not play PG (control group) and those that did (Pokémon group). Any days the Fitbit failed to collect data were not considered in the calculation of averages. For some participants, no step and/or distance data were recorded during the experiment. These subjects were not included in the comparison, thus producing the different sample sizes for the comparisons.

The Matlab software (R2018a, Natick, USA) was applied for all statistical analyses. Differences in means were calculated by using a 2-way ANOVA with the condition (control or Pokémon) and season as factors. It was anticipated that students would be walking less in the colder months than in the warmer ones. Normality of distributions was tested with a 1-sample ShapiroWilk test. The statistical significance threshold was set $a$ priori to $p=0.05$. Season was found not to have an interaction effect as shown in the results section. Thus, season was not considered as a controlling variable for further evaluations.

Because of the small sample size $(n<30)$, bootstrapping [17] was used to evaluate the confidence interval (CI) of the mean difference in average daily step count and travelled distance between the groups. A bootstrapped 2-sided $t$-test was also performed. For the resampling, 10 million combinations were employed: convergence was obtained. The samples were unmatched.

The effect size between distribution means was calculated by using Hedges' $g$. The cut-off thresholds were from Cohen's original $d$ thresholds [18-20]. Differences in groups for binary choices from the rPAR-Q responses were compared with a $\chi^{2}$ test.

\section{Ethical approval}

The research related to human use has complied with all the relevant national regulations and institutional policies, has followed the tenets of the Declaration of Helsinki, and has been approved by the Pennsylvania State University Institutional Review Board.

\section{Informed consent}

Informed consent has been obtained from all individuals included in this study.

\section{Results}

Overall, 27 students (14 male) participated in the 2 -week study. Demographics for each of the groups collected at the start of the study is shown in Table 1.
Table 1. Participant anthropometric data (mean $\pm S D$ )

\begin{tabular}{lcccc}
\hline Group & Female/n & $\begin{array}{c}\text { Age } \\
\text { (years) }\end{array}$ & $\begin{array}{c}\text { BMI } \\
\left(\mathrm{kg} / \mathrm{m}^{2}\right)\end{array}$ & $\begin{array}{c}\text { Resting } \\
\text { heart rate } \\
(\mathrm{bpm})\end{array}$ \\
\hline Control & $7 / 16$ & $20.6 \pm 2.2$ & $27.05 \pm 5.0$ & $76.0 \pm 18.0$ \\
Pokémon & $6 / 11$ & $21.5 \pm 4.6$ & $27.25 \pm 5.3$ & $76.4 \pm 7.2$ \\
\hline
\end{tabular}

$S D$ - standard deviation, BMI - body mass index

The subjects' age, body mass index, and resting heart rate act as a proxy as to their level of physical fitness prior to the study. Across these dimensions, no statistical difference was found between the 2 groups. This is taken to mean that neither group should have been more likely than the other to be more physically active. Students were not required to have prior experience with using Fitbits or playing PG to participate in the study.

Two factors were used as an indication of the Pokémon group's compliance of playing PG: the participant's change in level and change in experience points (XP) over the 2 weeks. All participants in this group started the game at level 1. Among the subjects that reported their starting and final values, the median change in level was 5 and the median change in XP was 37,830. No participant had 0 gain in XP, thus all were considered to have complied with the protocol.

A boxplot of the average daily step count for the control and Pokémon groups is shown in Figure 1. Both distributions were deemed normal from the ShapiroWilk test.

The means of the 2 distributions were compared by using a 2-factor ANOVA. Grouping on 'season' resulted in $p=0.685$, showing no significant difference due to season. The difference between the means was further compared with a bootstrapped $t$-test. No significant difference was observed between the means $(p=0.845)$. The distribution of the bootstrapped difference between means is shown in Figure 2. The 95\% CI on mean step difference (control-Pokémon) was [-2072, 2438] steps. The Hedges' $g$ effect size between the groups equalled 0.066 .

A boxplot of the average daily distance for the control and Pokémon groups is presented in Figure 3. Both distributions were deemed normal from the ShapiroWilk test.

The means of the 2 distributions were compared by using a 2-factor ANOVA. Grouping on 'season' resulted in $p=0.354$, showing no significant difference due to season. The difference between the means was further compared with a bootstrapped $t$-test. No significant difference was observed between the means $(p=0.528)$. The distribution of the bootstrapped dif- 


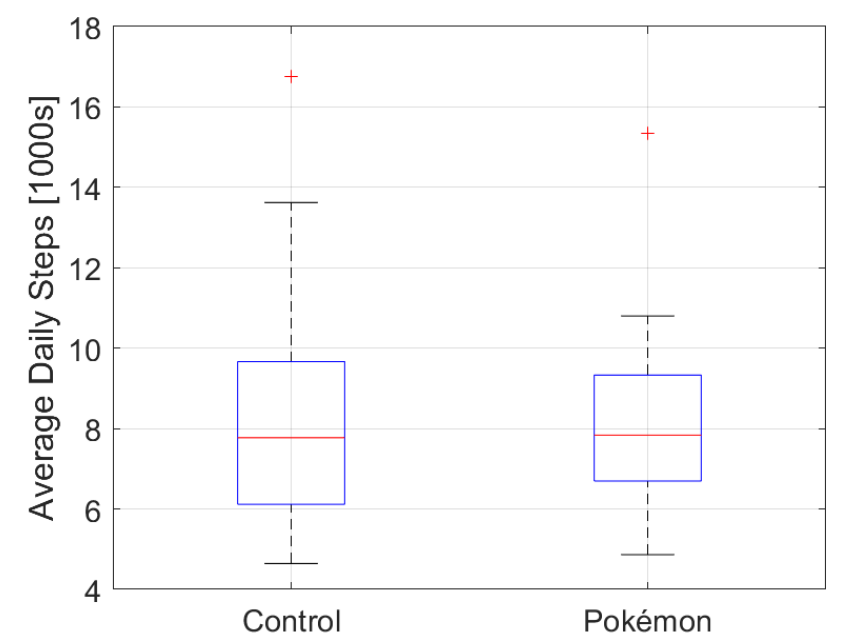

Figure 1. Boxplots of average daily step counts

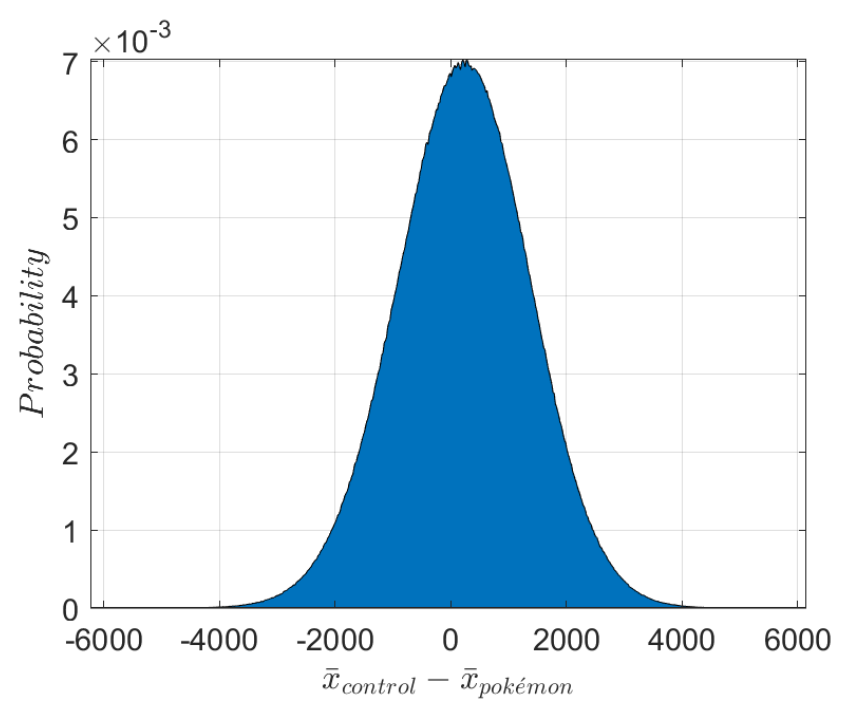

Figure 2. Probability density function of the bootstrapped difference between the means of the average daily step counts

ference between means is shown in Figure 4. The 95\% $\mathrm{CI}$ on mean distance difference (control-Pokémon) was $[-0.609,1.31] \mathrm{km}$. The Hedges' $g$ effect size between the groups equalled 0.24 .

Many participants in the study were physically active before the protocol began. This was revealed in the rPAR-Q administered prior to the study: $56 \%$ of the control group and $45 \%$ of the Pokémon group (not statistically different, $p=0.51$ by $\chi^{2}$ test) indicated that they engaged in moderate physical activity for at least 10 minutes daily.

\section{Discussion}

The aim of the study was to objectively quantify the impact of PG on physical activity, specifically step count

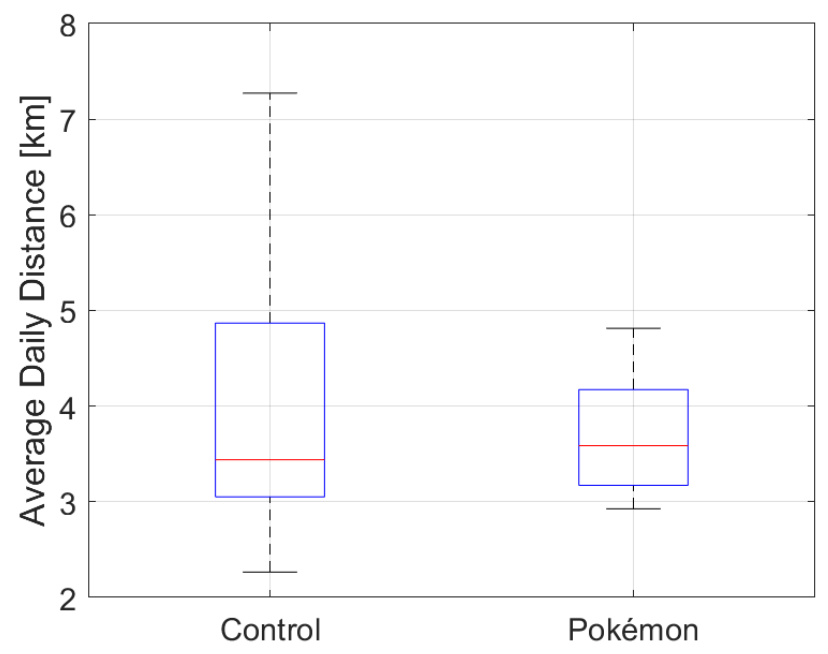

Figure 3. Boxplots of average daily distance

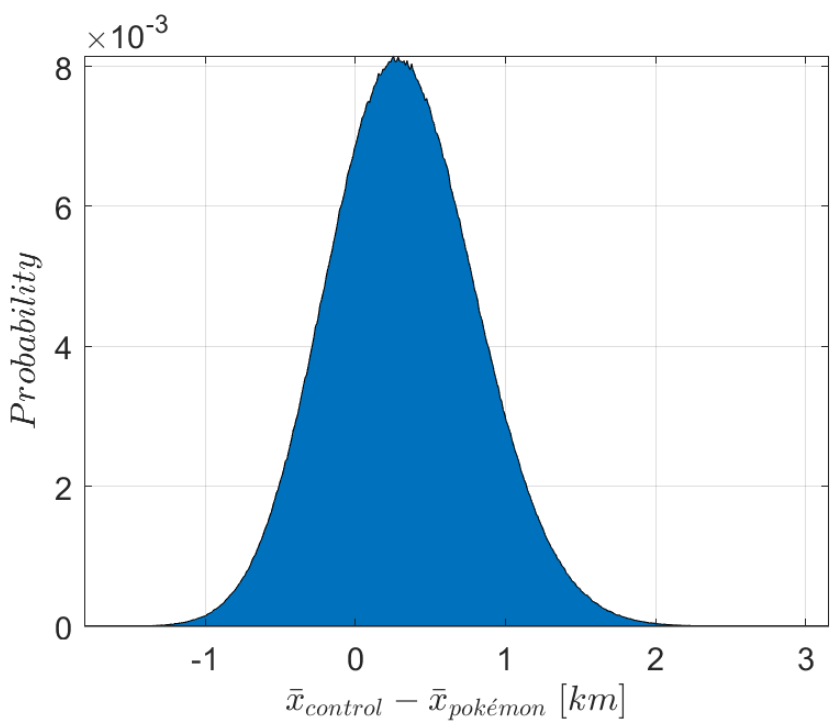

Figure 4. Probability density function of the bootstrapped difference between the means of the average daily distance

and distance travelled, among students within a local university setting. The results showed no statistically significant difference in the average daily step counts between the control and Pokémon participants ( $p=$ 0.845 ). Furthermore, no statistically significant difference was observed in the average daily distance travelled $(p=0.528)$. This implies that there was no significant difference in physical activity with respect to step counts or distance between students who played PG for 2 weeks and the control group. Additionally, when testing for interaction effects of season, there was no significant effect found (both $p>0.05$ ). The Hedges' $g$ values further demonstrate these small differences between groups. The effect size between the control and PG for the number of steps was 0.066, indicating very small to small effect. For distance, it 
was found to be 0.24 , indicating a small effect size. Bootstrapping the difference in means showed that the differences between the groups were small. The mean difference in daily step counts was revealed to be near zero, with symmetric CI. The mean daily distance and step count were actually less for the Pokémon players than the controls.

The official physical activity guidelines $[1,2]$ equate to approximately 8000-10,000 daily steps [21, 22]. Only about $21 \%$ of US adults meet these guidelines. Novel methods are routinely investigated to determine their effects on promoting physical activity, especially among students, whose sedentary behaviour appears to be increasing.

A complete physical activity routine should include aerobic exercise as well as resistance training [2]. Although PG cannot replace ordinary exercise as its benefits on cardio health are limited unless the players jog and walk briskly [6], getting inactive people to do even a small amount of physical activity is shown to provide greater population health gains even if the goal of 150 minutes a week is not achieved [23]. Participation in any leisure-time activity, even below the recommended level, is associated with a reduced risk of mortality and longer life expectancy [24], as well as decreasing lifetime medical expenditure [25]. A previous study found that playing active video games could be a tool to promote acute reduction of blood pressure in hypertensive patients with results like those of walking exercise [26].

It should be noted that the results could be due to interest in PG itself. Another study, conducted for a 3-month period, also found no statistically significant increase in activity during PG. It utilized self-report surveys of physical activity and revealed that $57.7 \%$ of the participants had stopped playing the game after 3 months. It was observed that players utilized the application for fun or as a pastime rather than for fitness purposes [27]. Another study findings were consistent with this in that the players' step intake increased for the first 5 weeks after downloading the application, and then returned to pre-installation levels [12]. These studies differ from the current study in that they applied self-reporting measures rather than researchers tracking the participants' activity levels. Another study found that while PG players spent more time outdoors than non-players, there was no statistically significant difference in physical activity [28]. It was conducted among a population similar to that in the present study, involving university students of both genders with a primary age range of 18-25 years [28].

One strength of this study is that Fitbits were used to objectively monitor and assess physical activity.
These allowed researchers to track the step counts of each participant without the use of self-reporting, which provided greater accuracy. A control group was employed in the study, which served to compare the activity levels of Pokémon players and non-players. The study utilized multiple cohorts, enabling researchers to compare results for various seasons throughout the academic year.

This study had some limitations. Among them, there was a small sample size. A smaller sample size decreases the statistical power of the study. However, bootstrapping was used to establish the CI. Another limitation is that the Fitbit tracker is worn on the wrist rather than the hip. This could potentially lead to miscounts in the numbers of steps taken, but previous studies applied wrist-based activity monitors [6]. Additionally, this study was conducted in 2-week cohorts; therefore, its results can only indicate short-term effects of PG on the college population.

This would possibly indicate that PG did not have an additional impact on physical activity in those who were already active. It would be consistent with the conclusion of another study that playing PG was most beneficial to players who were inactive prior to the investigation [28, 29].

\section{Conclusions}

PG was not found to promote physical activity, as measured by step count and distance, in the experimental group. Therefore, the game may not be the best tool to use in reducing sedentary behaviour among undergraduate students or be used as a starting point for inactive people to begin an active lifestyle. Active video games, however, could still have the potential to promote physical activity and a healthy lifestyle. Further research could be performed to see if a different active mobile game would prove to have a greater impact on students' activity in comparison with PG. Future studies could determine if active video gamers obtain an increase in physical activity in comparison with the general population. Finally, designers of mobile augmented reality games (e.g., PG, Harry Potter: Wizards Unite, Minecraft Earth) may wish to be more purposeful in integrating the incentive to walk into the game itself. These games each feature rewards for distance travelled, but future work needs to be done to balance the amount of the reward to truly increase users' activity. 


\section{Acknowledgements}

The authors would like to acknowledge the financial support from the Frank Franco Undergraduate Research Grant through Penn State Berks. They would also like to thank Justin Koop for his assistance in data collection.

\section{Disclosure statement}

No author has any financial interest or received any financial benefit from this research.

\section{Conflict of interest}

The authors state no conflict of interest.

\section{References}

1. Centers for Disease Control and Prevention. Physical Activity. 2014. Available from: https://www.cdc.gov/ physicalactivity/data/facts.htm.

2. Piercy KL, Troiano RP, Ballard RM, Carlson SA, Fulton JE, Galuska DA, et al. The physical activity guidelines for Americans. JAMA. 2018;320(19):2020-2028; doi: 10.1001/jama.2018.14854.

3. Vainshelboim B, Brennan GM, LoRusso S, Fitzgerald P, Wisniewski KS. Sedentary behavior and physiological health determinants in male and female college students. Physiol Behav. 2019;204:277-282; doi: 10.1016/j. physbeh.2019.02.041.

4. Racette SB, Deusinger SS, Strube MJ, Highstein GR, Deusinger RH. Weight changes, exercise, and dietary patterns during freshman and sophomore years of college. J Am Coll Health. 2005;53(6):245-251; doi: 10.3200/JACH.53.6.245-251.

5. Buckworth J, Nigg C. Physical activity, exercise, and sedentary behavior in college students. J Am Coll Health. 2004;53(1):28-34; doi: 10.3200/JACH.53.1.28-34.

6. Althoff T, White RW, Horvitz E. Influence of Pokémon Go on physical activity: study and implications. J Med Internet Res. 2016;18(12):e315; doi: 10.2196/jmir.6759.

7. Lindqvist AK, Castelli D, Hallberg J, Rutberg S. The praise and price of Pokémon GO: a qualitative study of children's and parents' experiences. JMIR Serious Games. 2018;6(1):e1; doi: 10.2196/games.8979.

8. Pokémon GO. Updates: Celebrating the first anniversary of Pokémon GO! 2017. Available from: http:// pokemongo.nianticlabs.com/en/post/anniversary2017.

9. Fountaine CJ, Springer EJ, Sward JR. A descriptive study of objectively measured Pokémon GO playtime in college students. Int J Exerc Sci. 2018;11(7):526-532.

10. Nigg CR, Mateo DJ, An J. Pokémon GO may increase physical activity and decrease sedentary behaviors. Am J Public Health. 2017;107(1):37-38; doi: 10.2105/ AJPH.2016.303532.

11. Dunning D, Heath C, Suls JM. Flawed self-assessment: implications for health, education, and the workplace. Psychol Sci Public Interest. 2004;5(3):69-106; doi: 10.1111/j.1529-1006.2004.00018.x.
12. Howe KB, Suharlim C, Ueda P, Howe D, Kawachi I, Rimm EB. Gotta catch'em all! Pokémon GO and physical activity among young adults: difference in differences study. BMJ. 2016;355:i6270; doi: 10.1136/bmj. i6270.

13. Ni MY, Hui RWH, Li TK, Tam AHM, Choy LLY, Ma $\mathrm{KKW}$, et al. Augmented reality games as a new class of physical activity interventions? The impact of Pokémon Go use and gaming intensity on physical activity. Games Health J. 2019;8(1):1-6; doi: 10.1089/g4h.2017. 0181.

14. Vosburg E, Zaichenko D, Knittle S, Sforzo G, Micale F, Crawford J, et al. Validity of the Fitbit Charge HR2 for measuring step count and heart rate during exercise. Int J Exerc Sci Conf Proc. 2018;9(6):132.

15. Burton E, Hill KD, Lautenschlager NT, ThøgersenNtoumani C, Lewin G, Boyle E, et al. Reliability and validity of two fitness tracker devices in the laboratory and home environment for older community-dwelling people. BMC Geriatr. 2018;18(1):103; doi: 10.1186/ s12877-018-0793-4.

16. Thomas S, Reading J, Shephard RJ. Revision of the Physical Activity Readiness Questionnaire (PAR-Q). Can J Sport Sci. 1992;17(4):338-345.

17. Hall P. Theoretical comparison of bootstrap confidence intervals. Ann Statist. 1988;16(3):927-953; doi: 10.1214/ aos/1176350933.

18. Lakens D. Calculating and reporting effect sizes to facilitate cumulative science: a practical primer for t-tests and ANOVAs. Front Psychol. 2013;4:863; doi: 10.3389/ fpsyg.2013.00863.

19. Hedges LV. Distribution theory for Glass's estimator of effect size and related estimators. J Educ Stat. 1981; 6(2):107-128; doi: 10.3102/10769986006002107.

20. Cohen J. Statistical power analysis for the behavioral sciences. New York: Routledge; 2013.

21. Tudor-Locke C, Craig CL, Brown WJ, Clemes SA, De Cocker K, Giles-Corti B, et al. How many steps/day are enough? For adults. Int J Behav Nutr Phys Act. 2011; 8:79; doi: 10.1186/1479-5868-8-79.

22. Tudor-Locke C, Leonardi C, Johnson WD, Katzmarzyk PT, Church TS. Accelerometer steps/day translation of moderate-to-vigorous activity. Prev Med. 2011;53(12):31-33; doi: 10.1016/j.ypmed.2011.01.014.

23. De Souto Barreto P. Global health agenda on non-communicable diseases: has WHO set a smart goal for physical activity? BMJ. 2015;350:h23; doi: 10.1136/bmj.h23.

24. Moore SC, Patel AV, Matthews CE, Berrington de Gonzalez A, Park Y, Katki HA, et al. Leisure time physical activity of moderate to vigorous intensity and mortality: a large pooled cohort analysis. PLoS Med. 2012;9(11): e1001335; doi: 10.1371/journal.pmed.1001335.

25. Nagai M, Kuriyama S, Kakizaki M, Ohmori-Matsuda K, Sone T, Hozawa A, et al. Impact of walking on life expectancy and lifetime medical expenditure: the Ohsaki Cohort Study. BMJ Open. 2011;1(2):bmjopen-2011000240; doi: 10.1136/bmjopen-2011-000240. 


\section{HUMAN MOVEMENT}

S. Schade et al., Pokémon Go did not increase physical activity

26. Da Silva TF, de Franca ACL, de Souza MF, Silva AS. A single session of active video game play promotes postexercise hypotension in hypertensive middle-aged subjects. Hum Mov. 2018;19(2):82-89; doi: 10.5114/hm. 2018.74063.

27. Wattanapisit A, Saengow U, Ng CJ, Thanamee S, Kaewruang N. Gaming behaviour with Pokémon GO and physical activity: a preliminary study with medical students in Thailand.PLoS One. 2018;13(6):e0199813; doi: 10.1371/journal.pone.0199813.

28. Wong FY. Influence of Pokémon Go on physical activity levels of university players: a cross-sectional study. Int J Health Geogr. 2017;16:8; doi: 10.1186/s12942-0170080-1.

29. Ma BD, Ng SL, Schwanen T, Zacharias J, Zhou M, Kawachi I, et al. Pokémon GO and physical activity in Asia: multilevel study. J Med Internet Res. 2018;20(6):e217; doi: 10.2196/jmir.9670. 